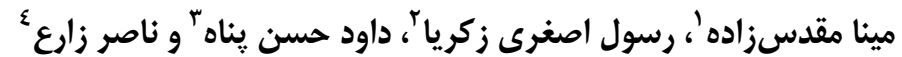

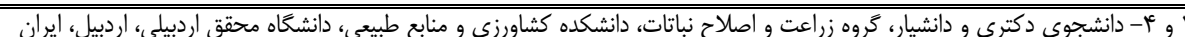

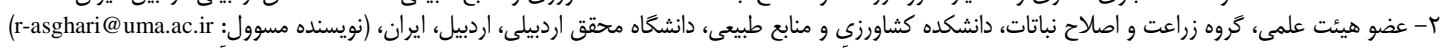

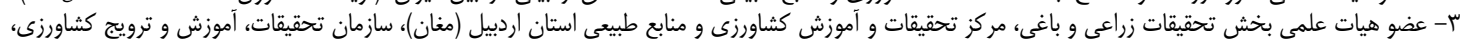
اردبيل، ايران

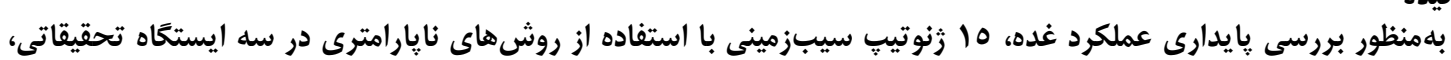

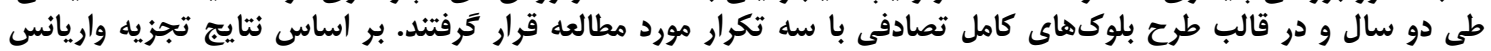

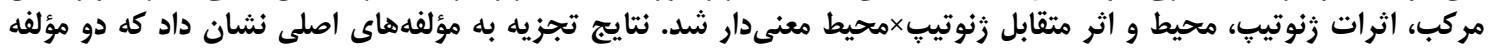

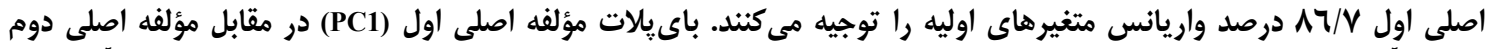

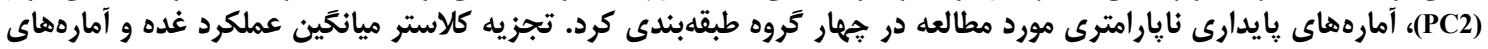

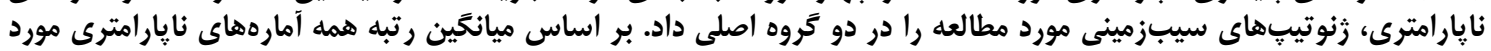

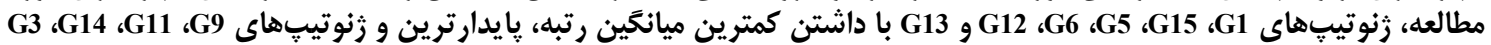

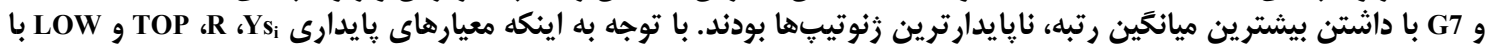

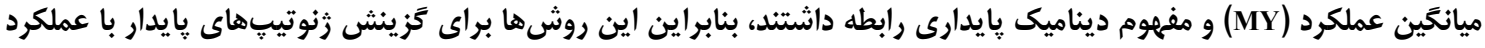

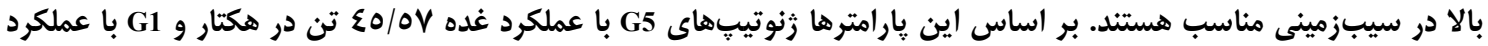

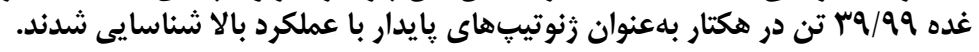

$$
\text { وازههاى كليدى: تجزيه پايدارى، تجزيه به مؤلفههاى اصلى، تجزيه كلاستر، سيبزمينى }
$$

اثرى ندارد. همجنين تجزيه و تحليل و و تفسير معيارهاى (باري

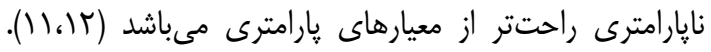

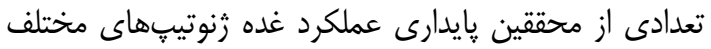

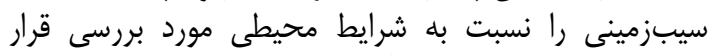

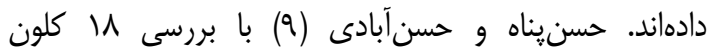

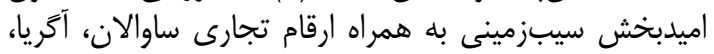

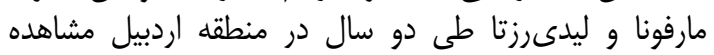

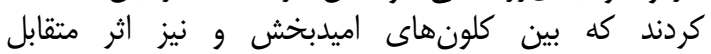

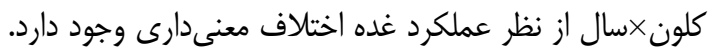

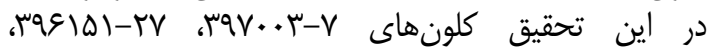

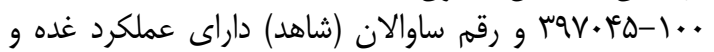

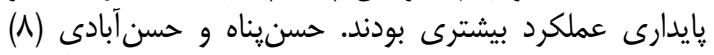

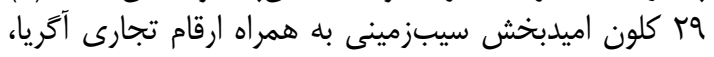

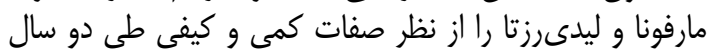

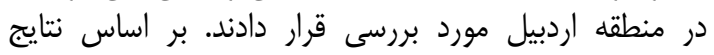

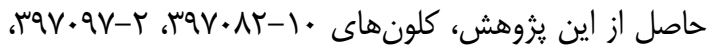

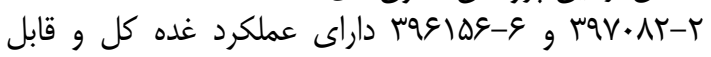

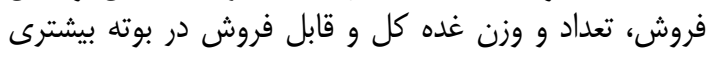

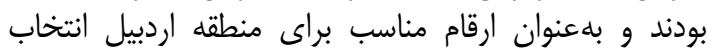

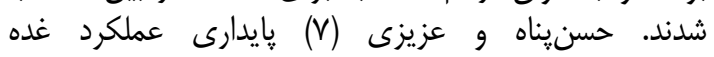

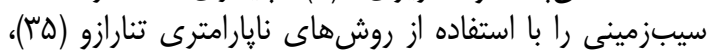

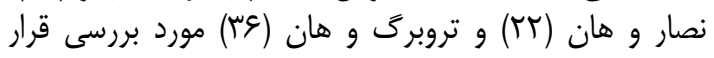

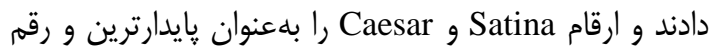

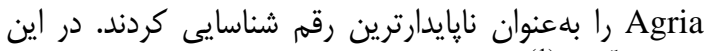
تحقيق آماره
مقدمه

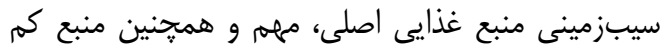

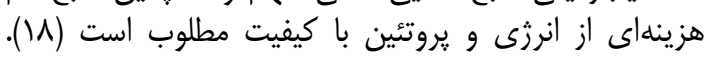

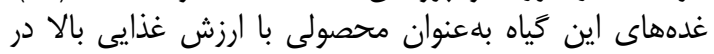

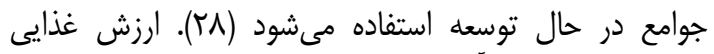

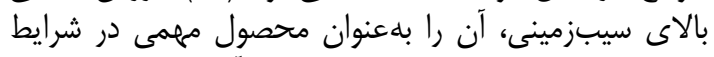

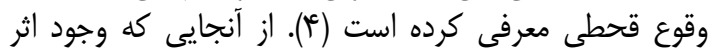

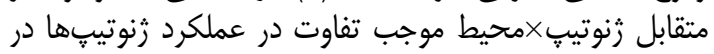

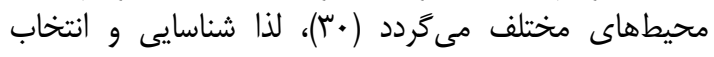

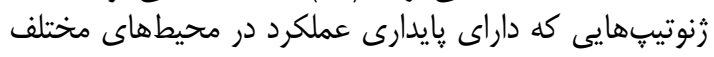

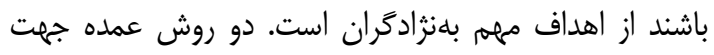

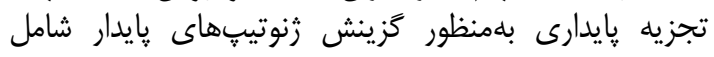

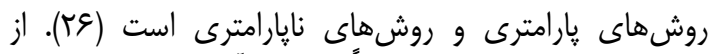

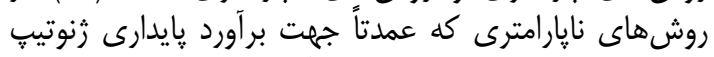

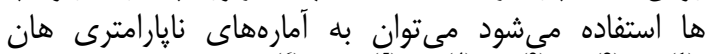

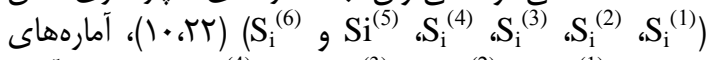
تنارازو

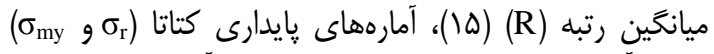

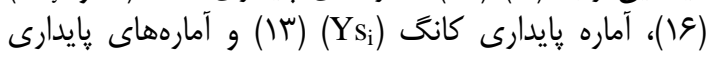

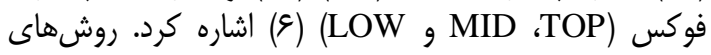

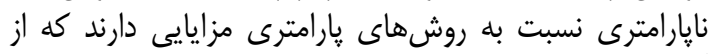

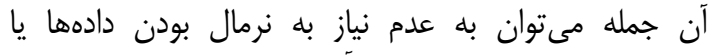

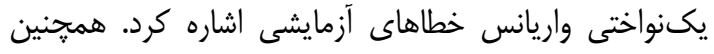

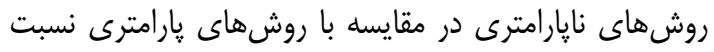

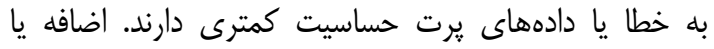

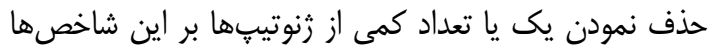




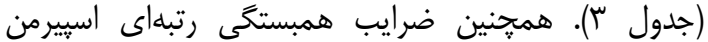

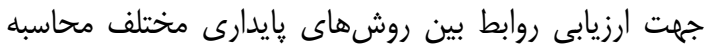

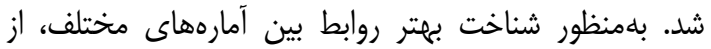

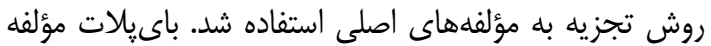

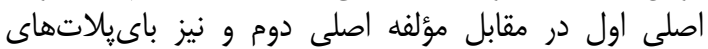

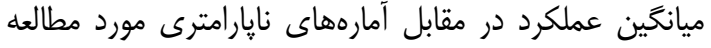

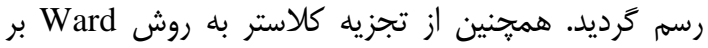

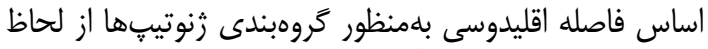

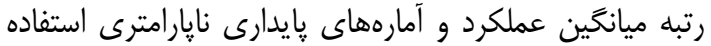

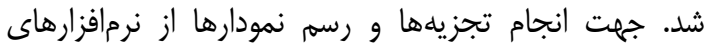
Minitab ، SAS

آماره عملكرد-يايدارى كانَى (YSi)

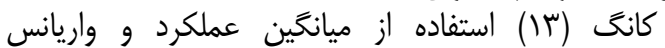

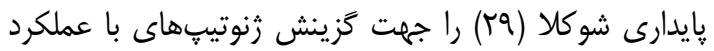

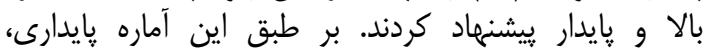

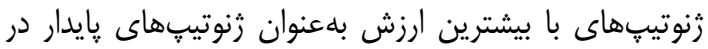
نظر كرفتله مي شوند.

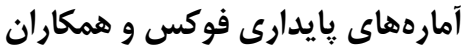

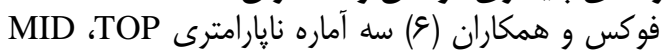

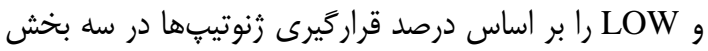

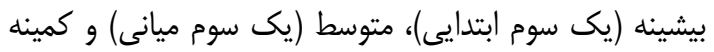

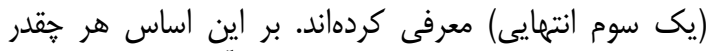

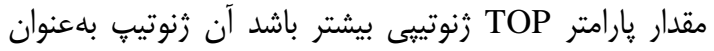

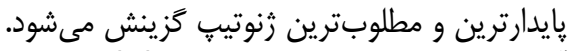

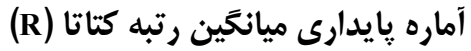

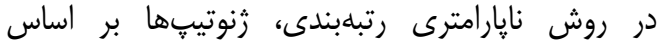

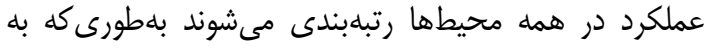

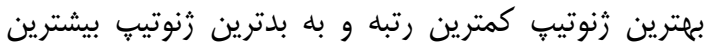

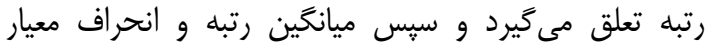

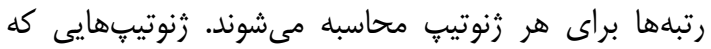

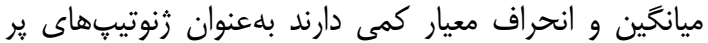
يتانسيل محسوب مىشوند (10).
تحقيق حاضر بلمنظور بررسى اثر متقابل زنوتيِلمحيط

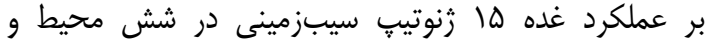

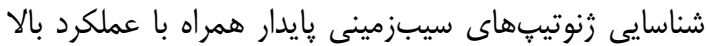

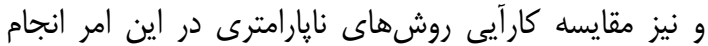
يذيرفت.

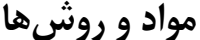

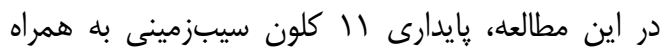
جهمار واريته (كايزر، لوستا، ساوالان و آكَريا) در طى دو دو سال

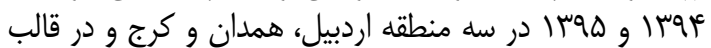

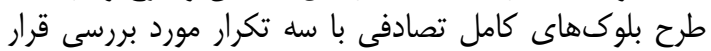

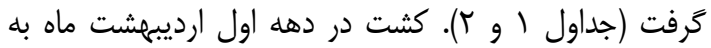

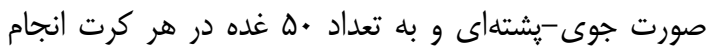

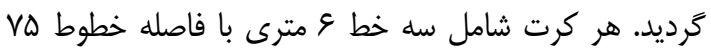

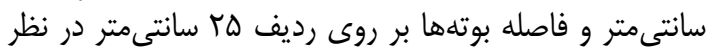

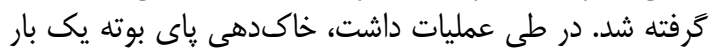

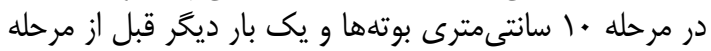

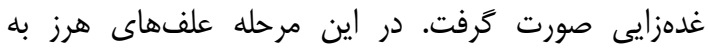

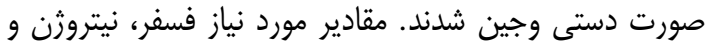

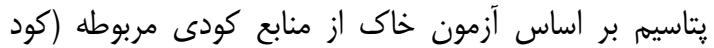

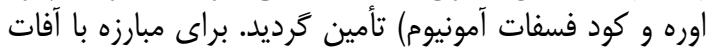

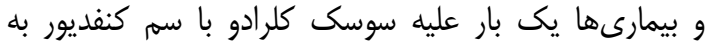

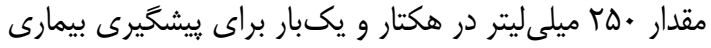

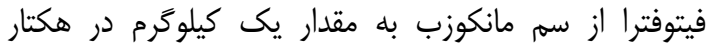

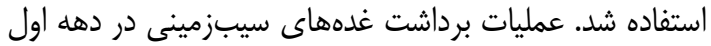

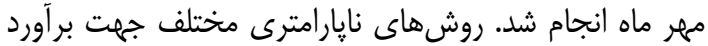

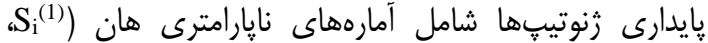

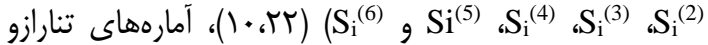
هي

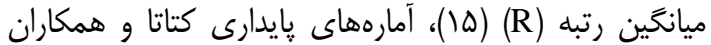
باري

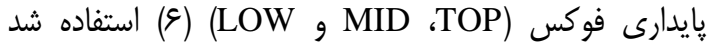

Table 1. The experimental sites and geographical characteristics of testing environments

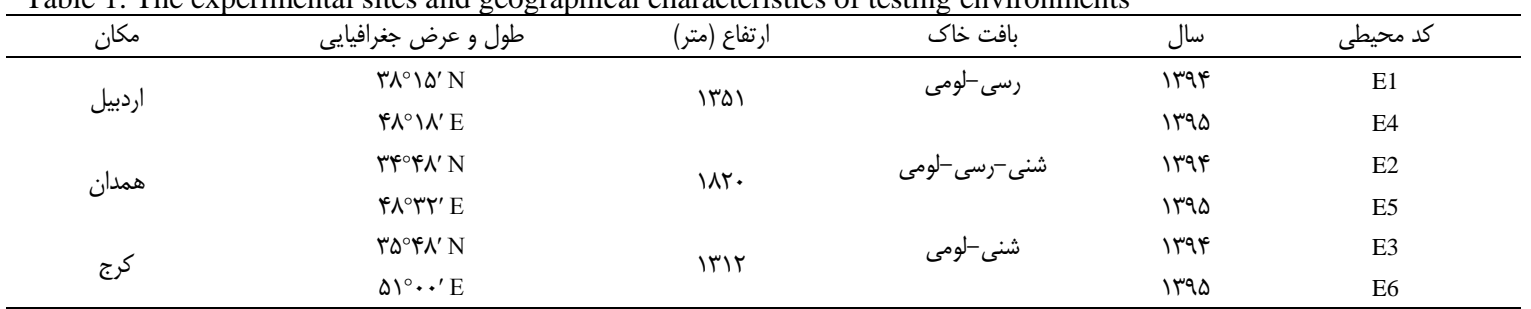


جدول r- كد زنوتيبى، والدين و ميانگين عملكرد غده ها زنوتيٍ سيبزمينى در شش محيط Table 2. Genotype code, parents and mean tuber yield of 15 potato genotypes in six environments

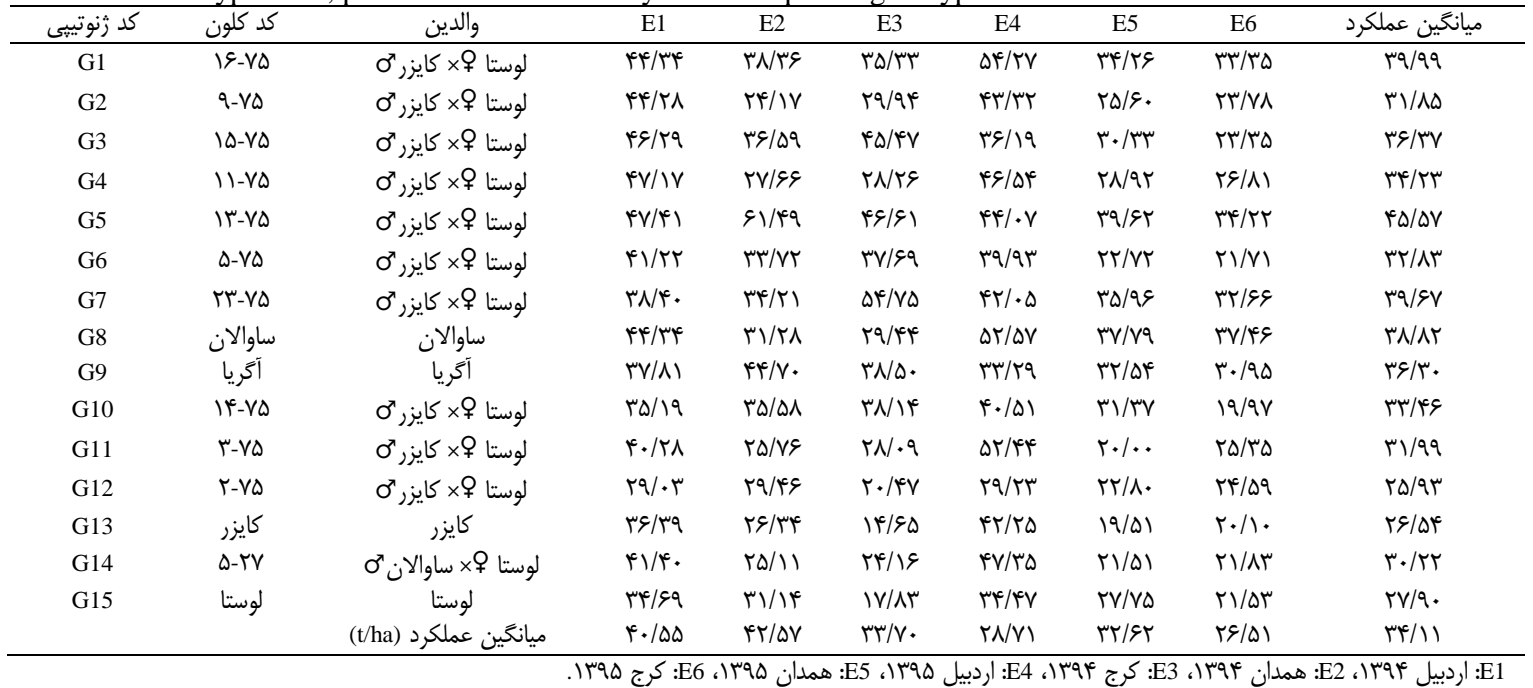

Table 3. Equations of nonparametric stability procedures

جدول س- فرمولهاى مربوط به آمارههاى نايار امترى

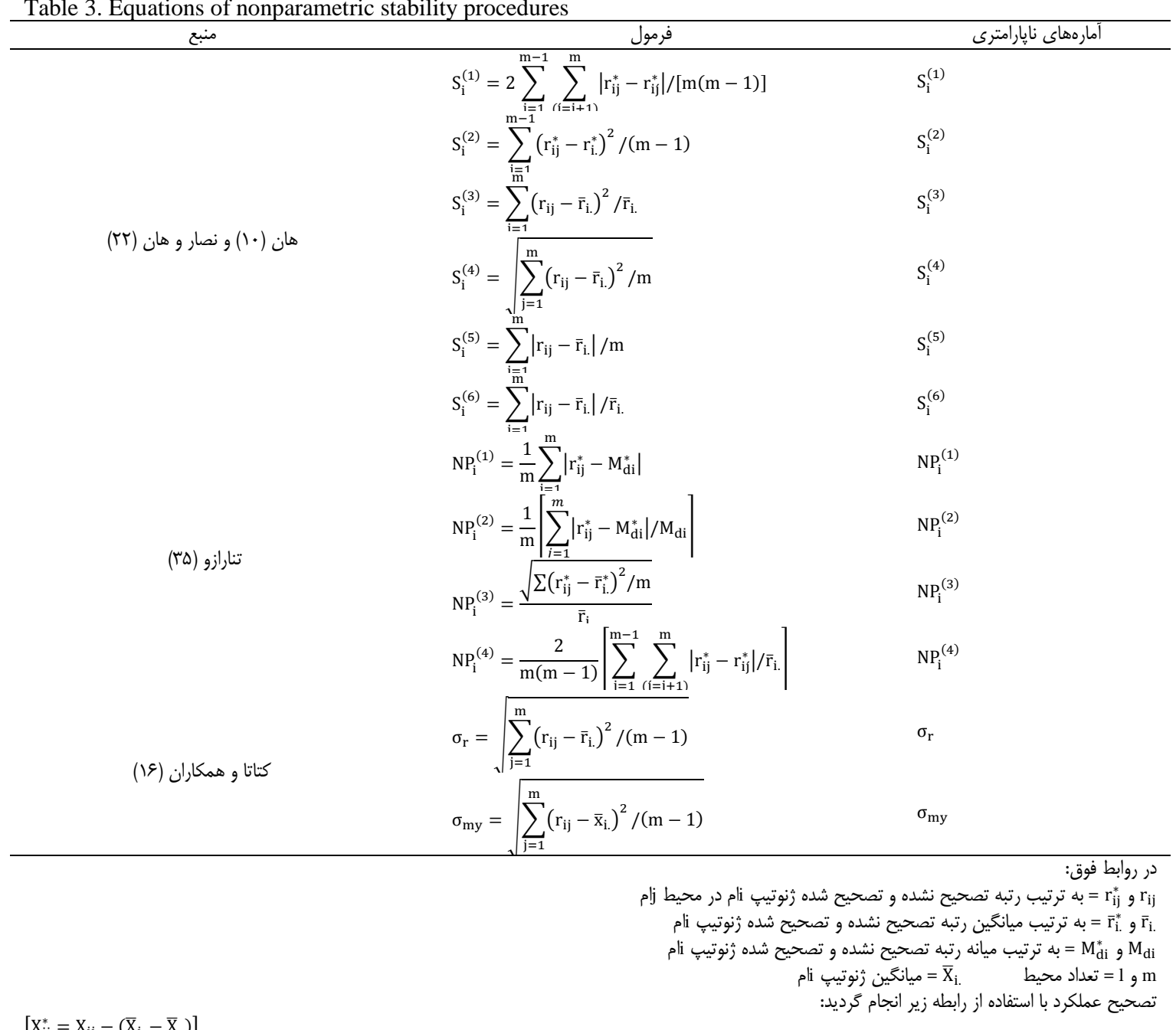


در سطح احتمال يك درصد معنىدار بود (جدول عأ). اثرات

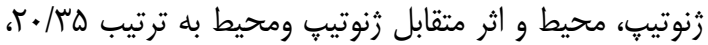

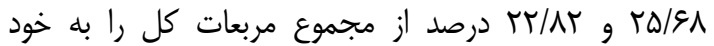

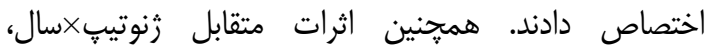

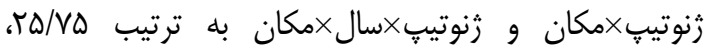

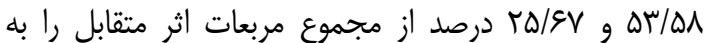

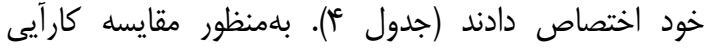

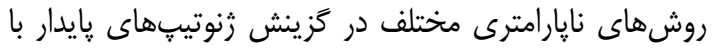

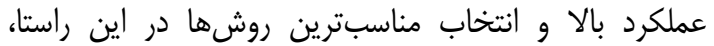

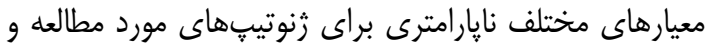

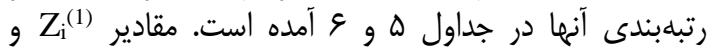

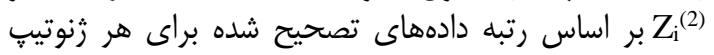

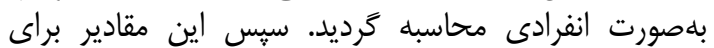

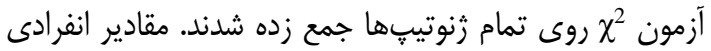

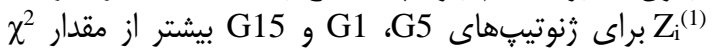

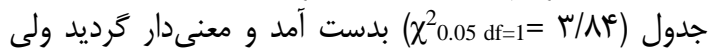
مقادير انفرادى

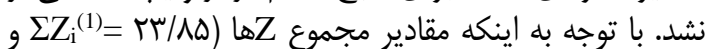
كمتر از مقدار بحرانى كاى اسكور جدول بان بان

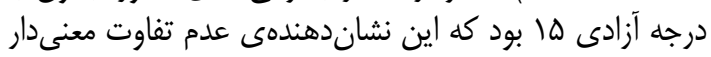

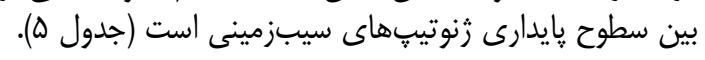

$$
\begin{aligned}
& \text { نصار و هان (ع) آزمون معنىدارى براى }
\end{aligned}
$$

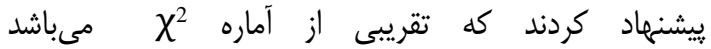

$$
\begin{aligned}
& :\left(\chi^{2}=\sum_{\mathrm{i}}^{\mathrm{l}} \mathrm{Z}_{\mathrm{i}}^{\mathrm{t}} \mathrm{t}=1,2\right) \\
& \mathrm{Z}_{\mathrm{i}}^{\mathrm{t}}=\left[\mathrm{S}_{\mathrm{i}}^{\mathrm{t}}-\mathrm{E}\left(\mathrm{S}_{\mathrm{i}}^{\mathrm{t}}\right)\right]^{2} / \mathrm{V}\left(\mathrm{S}_{\mathrm{i}}^{\mathrm{t}}\right)
\end{aligned}
$$$$
\text { در رابطه فوق E( }
$$

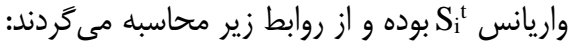$$
\mathrm{E}\left(\mathrm{S}_{\mathrm{i}}^{(1)}\right)=\frac{\mathrm{l}^{2}-1}{3 \mathrm{l}}
$$$$
\mathrm{E}\left(\mathrm{S}_{\mathrm{i}}^{(2)}\right)=\frac{\mathrm{l}^{2}-1}{12}
$$$$
\mathrm{V}\left(\mathrm{S}_{\mathrm{i}}^{(1)}\right)=\frac{\left(\mathrm{l}^{2}-1\right)\left[\left(\mathrm{l}^{2}-4\right)(\mathrm{m}+3)+30\right]}{45 \mathrm{l}^{2} \mathrm{~m}(\mathrm{~m}-1)}
$$$$
\mathrm{V}\left(\mathrm{S}_{\mathrm{i}}^{(2)}\right)
$$$$
=\frac{\left(1^{2}-1\right)\left[2\left(1^{2}-4\right)(m-1)+5\left(1^{2}-1\right)\right]}{360 m(m-1)}
$$

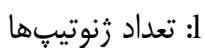
m تعداد محيطها

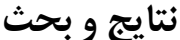

بر اساس نتايج جدول تجزيه مركب اثرات اصلى رنوتيب، سال و مكان و نيز اثرات متقابل زنوتيب در سال و در مكان أنران

جدول أ- تجزيه واريانس مركب عملكرد غده ها زنوتيب سيبزمينى در شش محيط

\begin{tabular}{|c|c|c|c|c|}
\hline$\%$ GE SS & $\% \mathrm{~T} \mathrm{SS}$ & ميانخين مربعات & درجه آزادى & منابع تغيير \\
\hline & 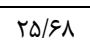 & $|\Lambda| N / \Lambda . * *$ & $\Delta$ & محيط (E) \\
\hline & & $9 \Delta \cdot / q^{* * *}$ & 1 & سال (Y) \\
\hline & & 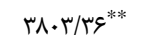 & r & مكان (L) \\
\hline & & $F \mid N / \Delta *^{* * *}$ & r & سال ×مكان (Y×L) \\
\hline & & TYT/TD & ir & خطاى اول (E1) \\
\hline & $r \cdot / \pi \omega$ & $\Delta \mid f / 99^{* * *}$ & 14 & رنوتي (G) \\
\hline & 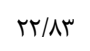 & $\| Q / \leftarrow \wedge^{* *}$ & v. & رنوتيب×محيط (G×E) \\
\hline$r \Delta / v \Delta$ & & $\mid \leftarrow N / V^{* * *}$ & 11 & رنوتي؟Xسال (G×Y) \\
\hline$\Delta \Psi / \Delta \Lambda$ & & $\mid Q f / \& V^{* *}$ & $r \Lambda$ & زنوتيب×مكان (G×L) \\
\hline \multirow[t]{4}{*}{$r \Delta / \overline{r V}$} & & $\Delta Q / 9 \Lambda$ & rs & زنوتيڤXسال ×مكان (G×Y×L) \\
\hline & & $r q / 94$ & 191 & خطاى دوم(E2) \\
\hline & & |r|gr & rq9 & كل - مل \\
\hline & & $r \cdot 109$ & & ضريب تغييرات (درصد) \\
\hline
\end{tabular}
Table 4. Combined analysis of variance of tuber yield (ton per ha) of 15 potato genotypes in six environments

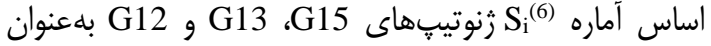

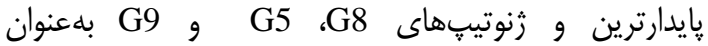

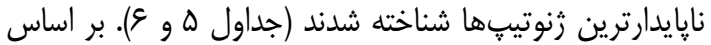
آمارههاى نايارامترى

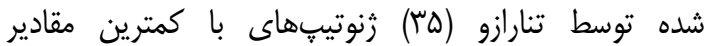

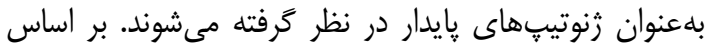

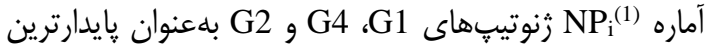

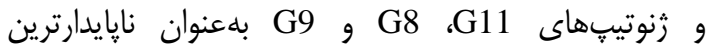

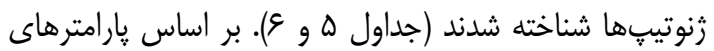

بر اساس آمارههاى يايدارى نايارامترى هان (+1) و نصار

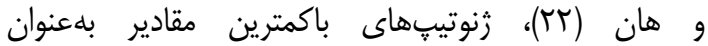

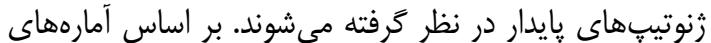
بان

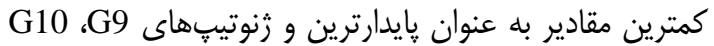

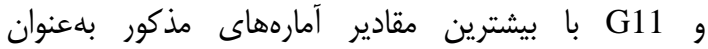

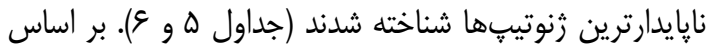
آماره زنوتيڤهاى G9، G8 و G3 نإيايدارترين بودند. همجنين بر 


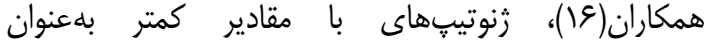

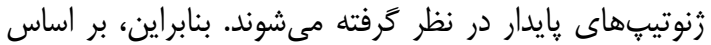

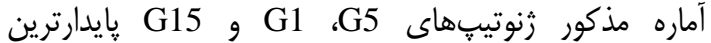

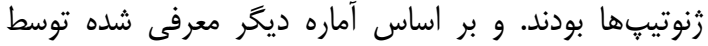

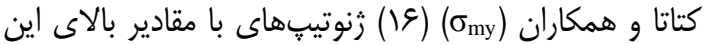

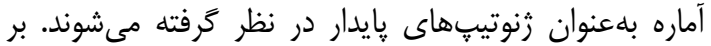
اين اساس ثنوتيڤهاى G5، G1 و G8 إيايدارترين بودند

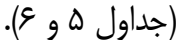
نباطور كلى بر اساس ميانخين رتبه همه آمارههاى

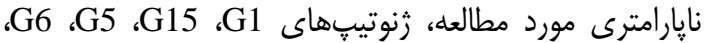

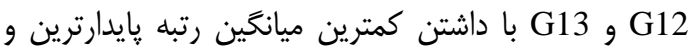

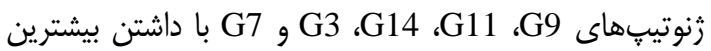

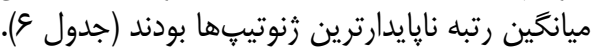

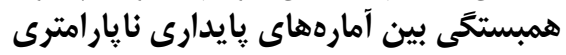

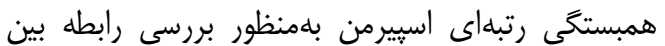

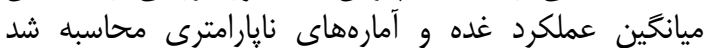

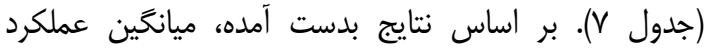

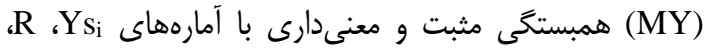

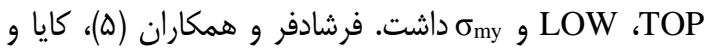

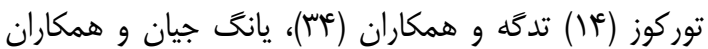

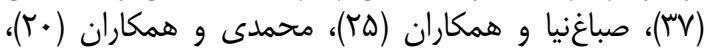

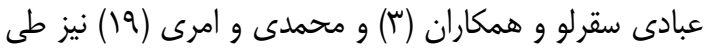

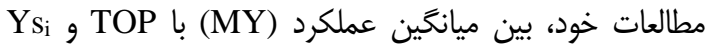

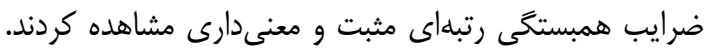

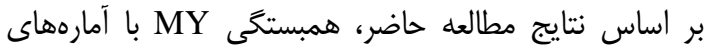
هايدارى

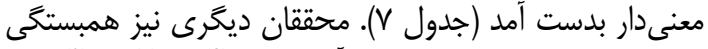
منفى و معنى دارى بين $\mathrm{MY}$ و آمارههاى

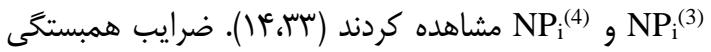

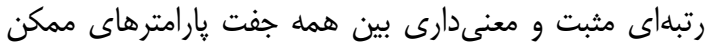

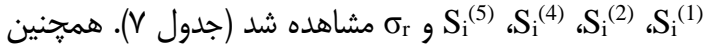
همبستكى مثبت و معنى دارى بين و

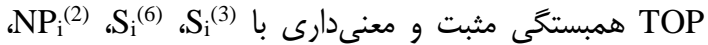

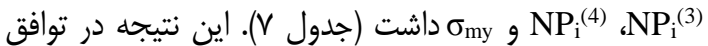
با نتايج ساير محققين است (VIVIVI)
منوتيبهاى G13 و $\mathrm{NP}^{(4)} \mathrm{NP}^{(2)}$

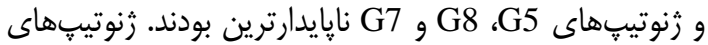

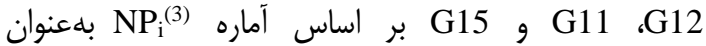

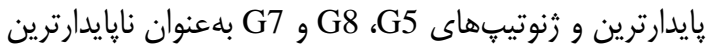

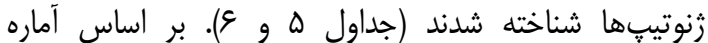
يايدارى كانگ (Ysi

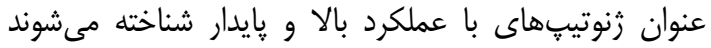

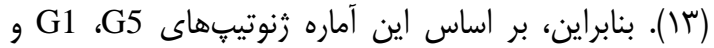

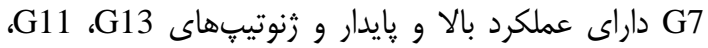

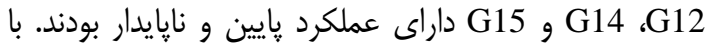
توجه به اينكه بر اساس آماره بايدارى كانغ (Ysi

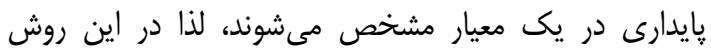

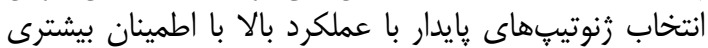

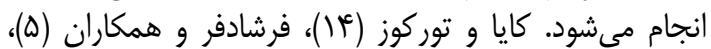

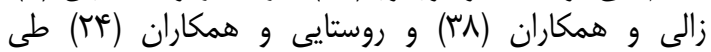

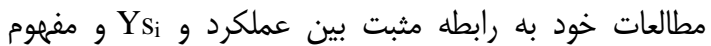

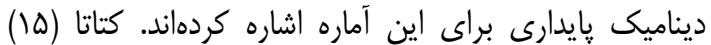

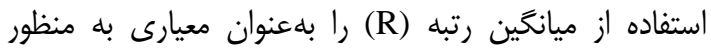

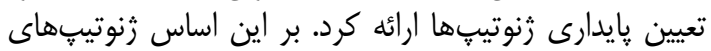

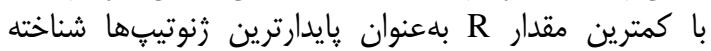

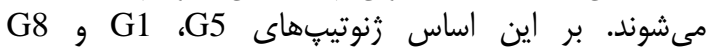

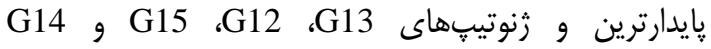

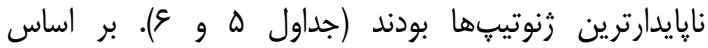

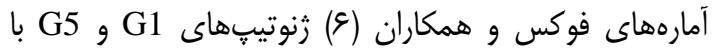

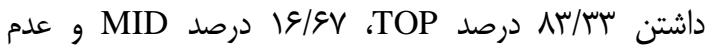

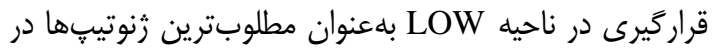

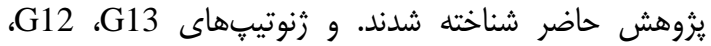
بر اساس آمارههاى مذكور بلعنوان نإيايدارترين

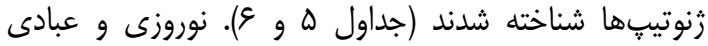

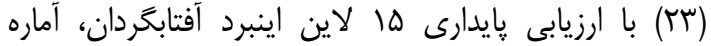

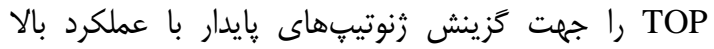

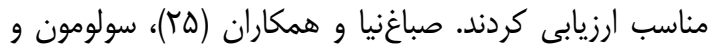

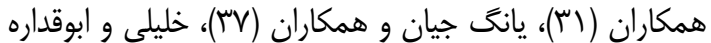

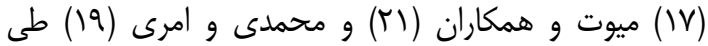

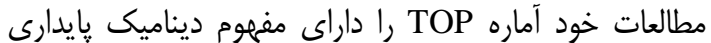

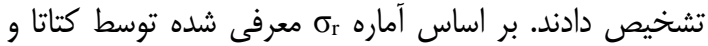




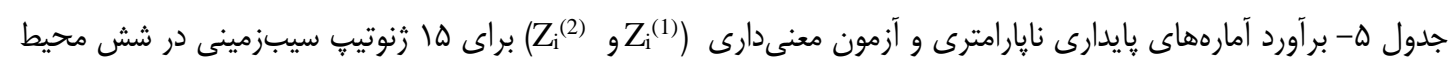
Table 5. Non-parametric stability parameters for yield and tests of non-parametric stability preceduers ( $\mathrm{Z}^{(1)}$ and $\mathrm{Z}_{\mathrm{i}}^{(2)}$ ) for 15 potato genotypes in six environments

\begin{tabular}{|c|c|c|c|c|c|c|c|c|c|c|c|c|c|c|c|c|c|c|c|c|}
\hline رنوتيب & MY (t/ha) & $\mathrm{Si}^{(1)}$ & $\mathrm{Z}_{\mathrm{i}^{(1)}}$ & $\mathrm{Si}^{(2)}$ & $\mathrm{Z}^{(2)}$ & $\mathrm{Si}^{(3)}$ & $\mathrm{Si}^{(4)}$ & $\mathrm{Si}^{(5)}$ & $\mathrm{Si}^{(6)}$ & $\mathrm{NP}_{\mathrm{i}}^{(1)}$ & $\mathrm{NP}_{\mathrm{i}}^{(2)}$ & $\mathrm{NP}_{\mathrm{i}}^{(3)}$ & $\mathrm{NP}^{(4)}$ & $\mathrm{YSi}_{\mathrm{Si}}$ & $\mathrm{R}$ & TOP & MID & LOW & $\sigma_{\mathrm{r}}$ & $\sigma_{\mathrm{my}}$ \\
\hline G1 & rq/१q & $T / T V$ & $r / 9 F^{*}$ & $r / \Lambda \gamma$ & $r / T V^{n s}$ & $\Delta / T V$ & $1 / \wedge$. & $1 / \pi r$ & $T / M \Lambda$ & $1 / \Delta$. & . & $.1 \Delta T$ &.$/ 11$ & $191 .$. & $r / q V$ & ה & $\mid g / g V$ & $\%$ & 1/9V & $r q / \Delta f$ \\
\hline G2 & $\Gamma / \Lambda \Delta$ & $r /$. & $\cdot \mid q)^{\mathrm{ens}}$ & $11 / 8$. & $\cdot / V Y^{n s}$ & $s / 4 \varphi^{\circ}$ & $r / 11$ & ת & $1 / D F$ & $r / I V$ & $\cdot / r v$ &.$/ 4 \varphi^{4}$ &.$/ 1 F$ & $r / \cdot$. & १/.. & $19 / 9 V$ & $98 / 9 V$ & $19 / 9 V$ & $r / F i$ & TA/TS \\
\hline G3 & r & $r / 9$. & $\cdot / 1 \cdot{ }^{\text {ns }}$ & $\mid f / V$. & $\cdot / \pi^{\mu_{\mathrm{ns}}}$ & $11 / \mu 1$ & $\Gamma / \omega$. & $r / I V$ & r/ar & $r / q V$ &.$/ 9 V$ &.$/ 9 T^{c}$ & 每 & $\mid r / \cdot$. & $s / 0$. & $\Delta . /$. & ת & $\mid \& / 9 V$ & $r / A r$ & $r T / q F$ \\
\hline G4 & r & F/N & $\cdot / \mathcal{F}^{\mathrm{N}} \lambda^{\mathrm{ns}}$ & $11 / r$. & $\cdot|\Lambda|^{\mathrm{ns}}$ & $N / \cdot$ & $r / .9$ & $r / 9 V$ & $r / r q$ & $\mu / .$. & T Tא/. & $\cdot / \Delta \omega$ &.$/ \mathrm{V}$ & $N / \cdot \cdot$ & $\mathrm{V} / \cdot \cdot$ & זسז/ & $\Delta . /$. & $19 / 9 \mathrm{~V}$ & 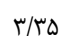 & $r . / .1$ \\
\hline G5 & $\Gamma \Delta / \Delta V$ & 1/9 & $\varepsilon / R \mu^{*}$ & $r / W V$ & $r / T)^{\mathrm{ns}}$ & $N / 99$ & $1 / w V$ & $1 / T \Lambda$ & $r / \Delta F$ & ת r & T/Tr & $1 / 99$ & .191 & $\mathrm{~N} / \cdot \cdot$ & $r / I V$ & ( & $19 / 9 V$ &. & $1 / 94$ & $F V / \Delta q$ \\
\hline G6 & r & $r / T V$ & $1 / 9 y^{n s}$ & $V / W V$ & $1 / N Y^{\mathrm{ns}}$ & $F / \pi F$ & $r / \Delta F$ & $r / \Delta$. & $1 / 94$ & $\mu / .$. & זr/. &.$/ 4 q$ & 西 & $8 / .$. & $q / 1 V$ & $\%$ & $\Delta . /$. & $\Delta \cdot / \cdot$. & $r / v q$ & $r q / . V$ \\
\hline G7 & $r q / q v$ & $\% /$. & $. / 9)^{\mathrm{cns}}$ & $1.19 \mathrm{~V}$ & . $/ 9 \mathrm{~F}^{\mathrm{ns}}$ & 1.1. & $r / Q 1$ & $r / q V$ & $r / .$. & $\varphi /$. & $\cdot / \Lambda$ & $\cdot / A r$ & $\cdot / \mu$ & $\mid Q / \cdot$. & ת & $\Delta \cdot /$. & $\Delta .1$. & $\%$ & $r / T V$ & $\mathrm{rV} / \mathrm{Vq}$ \\
\hline G8 & rN/AT & $\%$ & $. / 9)^{\mathrm{cns}}$ & $11 / 4 V$ & $\cdot / V \Delta^{\mathrm{ns}}$ & سז/Wו & $r / . q$ & $r / v \Lambda$ & $\Gamma / \Lambda \Delta$ & r & $1 / 4 x$ & $1 / 11$ &.$/ 4$. & $1 \% \%$ & r/r & $99 / 9 \mathrm{~V}$ & זس/ &.$/$. & $r / r q$ & rV/ar \\
\hline G9 & . & $\Delta / r$. &.$/ \cdot r_{\text {ns }}$ & $19 / \wedge V$ &.$/ . r^{\mathrm{ns}}$ & $1 \% / 9$. & $r / \cdot V$ & $r / \Delta S$ & $r / r$. & $\% / \cdot$ & $\cdot / \Lambda$ & $\cdot / N^{4}$ & $\cdot / T V$ & $11 / \cdot$. & $s / 9 V$ & $99 / 9 \mathrm{~V}$ & $\mid g / 9 V$ & $\mid s / 9 V$ & $F / 49$ & rT/VG \\
\hline G10 & ع & $\Delta / \cdot \cdot$ & $\cdot / .^{\mathrm{ns}}$ & $I V / r V$ &.$/ . r^{\mathrm{ns}}$ & $q / \Lambda \mu$ & $\Gamma / \Lambda$. & $\Gamma / \Delta$. & T/r & $\Gamma / \Lambda \mu$ &.$/ 4 \alpha$ &.$/ 4 V$ &.$/ N V$ & $\mathrm{~V} / \cdot \cdot$ & N/Ar & אזr/אr & س & r & $F / I V$ & $T V / r$. \\
\hline G11 & ५ /q9 & $\Delta / \cdot V$ & $\cdot 1 \cdot 1^{\mathrm{ns}}$ & $|V| \cdot V$ &.$/ . \mathrm{ens}^{2}$ & $9 / 14$ & $r / v V$ & س & $r / l f$ & $r / \Delta$. &.$/ 4 V$ & $\cdot / \Delta F$ &.$/ 19$ & $-r /$. & $q / r$ & $19 / 9 V$ & עr/ & $\Delta \cdot / \cdot \cdot$ & $r / N$ & $r \Delta / 19$ \\
\hline G12 & ro/qu & $r / T r$ & $1 / 9 Y^{n s}$ & s/av & $1 / 9 \Lambda^{\mathrm{ns}}$ & r/qY & $r / 4)$ & $r / N V$ & $1 / 1$. & $r / \Lambda r$ & Tr & r & 少 & $-1 / \cdot$ & $11 / \lambda r$ & $\%$ & r & $99 / 9 \mathrm{~V}$ & $r / q F$ & $\mid 0 / 99$ \\
\hline G13 & TS/QF & $r / T V$ & $1 / 9 V^{n s}$ & $V / \Delta$. & $1 / A)^{\mathrm{ns}}$ & $r /$. & $r / \omega$. & $r / I V$ & $1 / \cdot c^{c}$ & $r / q V$ & $\cdot / T \Lambda$ &.$/ \mu_{F}$ &.$/ T$ & $-\kappa /$. & $I r / \Delta$. &.$/$. & $\mid g / 9 V$ & 愐 & $T / M^{C}$ & $\mid Q / G T$ \\
\hline G14 & $r \cdot / r$ & $\varphi / \Lambda$. & $.1 . \mathrm{rns}^{\mathrm{ns}}$ & $18 / 4$. & $\cdot 1 \cdot \mathrm{v}^{\mathrm{ns}}$ & $N / r$. & $r / v$. & r & $r / \cdot$. & ת r &.$/ 4 q$ & תז/. &.$/ 19$ & $-1 / \cdot$ & $1 . \%$ & $\mid s / 9 V$ & $\mid \varepsilon / 9 V$ & $99 / 9 \mathrm{~V}$ & $F / \cdot \Delta$ & $T r / \Delta T$ \\
\hline \multirow[t]{2}{*}{ G15 } & $r V / q$. & $r / A^{E}$ & $F / \mathcal{F}^{*}$ & $r / q V$ & $r / V Y^{\mathrm{ns}}$ & $r / 1$. & $r / r$ & $1 / 19$ &.$/ 99$ & $r / q V$ & $\cdot / r \Lambda$ & . &.$/ T$ & $1 / \cdot \cdot$ & $11 / \Delta \mu$ & $\cdot /$. & זس/ & $99 / 9 \mathrm{~V}$ & T/R & $I V / V V^{e}$ \\
\hline & & $\begin{array}{c}\Sigma Z_{\mathrm{i}}^{(1)}=r \mu / \Lambda \triangle \\
\text { Test } \\
\text { Statistics }\end{array}$ & & $\Sigma Z_{i}^{(2)}=I N / T$. & & & & & & & & & & & & & & & & \\
\hline $\begin{array}{l}\text { Mean } \\
\text { Yield }\end{array}$ & & $\mathrm{E}\left(\mathrm{S}_{\mathrm{i}}^{(1)}\right)$ & & $\mathrm{E}\left(\mathrm{S}_{\mathrm{i}^{(2)}}\right)$ & & $\operatorname{Var}\left(\mathrm{Si}^{(1)}\right)$ & & $\operatorname{Var}\left(\mathbf{S}^{(2)}\right)$ & & $x^{2} z 1, z 2$ & & $\chi_{\text {sum }}^{2}$ & & & & & & & & \\
\hline$r \varphi / 11$ t/ha & & r/qVV & & 1N/999 & & $1 / F M$ & & $99 / .99$ & & $r / \Lambda F$ & & $r r / V$ & & & & & & & & \\
\hline
\end{tabular}


جدول 9- رتبه ها رنوتيب سيبزمينى براى آمارههاى پايدارى نايارامترى

Table 6. Ranks of 15 potato genotypes for non-parametric stability parameters

\begin{tabular}{|c|c|c|c|c|c|c|c|c|c|c|c|c|c|c|c|c|c|c|c|}
\hline رنوتيٍ & MY & $\mathrm{S}_{\mathrm{i}}^{(1)}$ & $\mathrm{S}_{\mathrm{i}}^{(2)}$ & $\mathrm{Si}_{\mathrm{i}}^{(3)}$ & $\mathrm{S}_{\mathrm{i}}^{(4)}$ & $\mathrm{Si}^{(5)}$ & $\mathrm{S}_{\mathrm{i}}{ }^{(6)}$ & $\mathrm{NP}_{\mathrm{i}}^{(1)}$ & $\mathrm{NP}_{\mathrm{i}}^{(2)}$ & $\mathrm{NP}_{\mathrm{i}}^{\left({ }^{(3)}\right.}$ & $\mathrm{NP}_{\mathrm{i}}{ }^{(4)}$ & $\mathrm{Ys}_{\mathrm{i}}$ & $\mathrm{R}$ & TOP & MID & LOW & $\sigma_{\mathrm{r}}$ & $\sigma_{\mathrm{my}}$ & ميانكين رتبه \\
\hline G1 & r & r & $r$ & Q & $r$ & $r$ & $\wedge$ & 1 & 8 & $\wedge$ & $v$ & r & r & 1 & r & 1 & r & r & $\Gamma / \Gamma \Delta$ \\
\hline G2 & 11 & 9 & 1. & 9 & 1. & $\Delta$ & f & $r$ & $\Delta$ & 9 & f & 1. & 9 & $\Delta$ & 1 & $r$ & 1. & 1. & $g / N F$ \\
\hline G3 & $\Delta$ & $\wedge$ & 11 & זו & 11 & 9 & 11 & $\Delta$ & 9 & 11 & 9 & $\Delta$ & $\Delta$ & r & $r$ & $r$ & 11 & $\Delta$ & $\mathrm{V} / \mathrm{VI}$ \\
\hline G4 & v & $v$ & $\wedge$ & $v$ & $\wedge$ & v & 9 & $r$ & 9 & 1. & 9 & v & $v$ & r & $r$ & $r$ & 1 & v & s/rq \\
\hline G5 & 1 & 1 & 1 & 9 & 1 & 1 & $1 f$ & f & $\pi$ & 10 & r & 1 & 1 & 1 & r & 1 & 1 & 1 & $F / V G$ \\
\hline G6 & 9 & r & 9 & F & 9 & 9 & $\Delta$ & $r$ & r & f & $r$ & 9 & 1. & 9 & $r$ & $c^{c}$ & 9 & 9 & $\Delta / r q$ \\
\hline G7 & $r$ & 8 & $v$ & $\pi$ & v & $v$ & Ir & $v$ & 1. & Ir & 11 & r & r & r & $r$ & 1 & v & r & SIAT \\
\hline G8 & c & 9 & 9 & 16 & 9 & $\wedge$ & 10 & 1 & 11 & 18 & $\pi$ & ז & $r$ & $r$ & $r$ & 1 & 9 & $r$ & $\mathrm{~V} / \mathrm{VI}$ \\
\hline G9 & 9 & Ir & 10 & 10 & 10 & $\pi$ & r & $v$ & 1. & Ir & 1. & 9 & 9 & r & r & r & 10 & 8 & $9 / \Delta \Gamma$ \\
\hline G10 & $\wedge$ & 1. & 15 & 11 & 15 & 11 & 1. & 8 & $\wedge$ & $v$ & 9 & $\wedge$ & $\wedge$ & r & r & r & 15 & $\wedge$ & N/QT \\
\hline G11 & 1. & 11 & r & 1. & r & 1. & v & 9 & v & 9 & $\wedge$ & זו & 11 & $\Delta$ & $r$ & f & r & 11 & $q / T F$ \\
\hline G12 & 10 & $\Delta$ & r & r & r & r & $r$ & 9 & r & 1 & r & ir & r & 8 & r & $\Delta$ & r & 15 & $\Delta / r \Delta$ \\
\hline G13 & 15 & $\Delta$ & $\Delta$ & r & $\Delta$ & r & r & $\Delta$ & 1 & r & 1 & 14 & 15 & 9 & f & 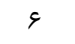 & $\Delta$ & 10 & $\Delta / v 1$ \\
\hline G14 & ir & 9 & ir & $\wedge$ & ir & 1. & 8 & f & r & $\Delta$ & $\Delta$ & ir & ir & $\Delta$ & r & $\Delta$ & ir & ir & V/qF \\
\hline G15 & r & $r$ & r & 1 & r & $r$ & 1 & $\Delta$ & 1 & $r$ & 1 & 11 & זו & 9 & $r$ & $\Delta$ & r & rו & $r / 09$ \\
\hline
\end{tabular}




\begin{tabular}{|c|c|c|c|c|c|c|c|c|c|c|c|c|c|c|c|c|c|}
\hline & MY & $\mathrm{Ys}_{\mathrm{i}}$ & $\mathrm{R}$ & $\mathrm{S}_{\mathrm{i}^{(1)}}^{(1)}$ & $\mathrm{S}_{\mathrm{i}}^{(2)}$ & $\mathrm{S}_{\mathrm{i}}^{(3)}$ & $\mathrm{Si}_{\mathrm{i}}^{(4)}$ & $\mathrm{S}_{\mathrm{i}}^{(5)}$ & $\mathrm{S}_{\mathrm{i}}{ }^{(6)}$ & $\mathrm{NP}_{\mathrm{i}}^{(1)}$ & $\mathrm{NP}_{\mathrm{i}}^{(2)}$ & $\mathrm{NP}_{\mathrm{i}}{ }^{(3)}$ & $\mathrm{NP}^{(4)}$ & TOP & MID & LOW & $\sigma_{\mathrm{r}}$ \\
\hline $\mathrm{Ysi}_{\mathrm{Si}}$ &.$/ 90^{* *}$ & & & & & & & & & & & & & & & & \\
\hline $\mathrm{R}$ & $\cdot / 9 \Lambda^{* *}$ & $\cdot / 99^{* *}$ & & & & & & & & & & & & & & & \\
\hline $\mathrm{S}_{\mathrm{i}}{ }^{(1)}$ &.$/ 11$ & $\cdot / T V$ &.$/ T$ & & & & & & & & & & & & & & \\
\hline $\mathrm{S}_{\mathrm{i}}^{(2)}$ & $\cdot 1 \cdot 1$ & $\cdot|r|$ &.$/ \cdot V$ &.$/ 9 * *$ & & & & & & & & & & & & & \\
\hline $\mathrm{S}_{\mathrm{i}}^{(3)}$ & $-\cdot / \wedge \varsigma^{* *}$ &.$- / v^{* * *}$ &.$- / \wedge v^{* *}$ &.$/ T \Delta$ & $\cdot / T V$ & & & & & & & & & & & & \\
\hline $\mathrm{Si}^{(4)}$ & $\cdot 1 \cdot 1$ & $\cdot|r|$ &.$/ \cdot V$ &.$/ 9 q^{* * *}$ & $1 / . . * *$ & $\cdot / T V$ & & & & & & & & & & & \\
\hline $\mathrm{S}_{\mathrm{i}}^{(5)}$ & $\cdot$ & $. / 1)^{c}$ & .1 .4 &.$/ 90^{* * *}$ &.$/ 99^{* *}$ & ع &.$/ 99^{* *}$ & & & & & & & & & & \\
\hline $\mathrm{S}_{\mathrm{i}^{(6)}}$ &.$- / g r^{*}$ & -.10 &.$-|g|^{*}$ &.$|q|^{* * *}$ & $\cdot \mid \& \phi^{* * *}$ & $\cdot / M^{* *}$ & $\cdot \mid \& \phi^{* * *}$ & $\cdot /\left.M\right|^{* *}$ & & & & & & & & & \\
\hline $\mathrm{NP}_{\mathrm{i}}^{(1)}$ & .1 .9 &.$/ 1 F$ & .1 .9 & $\cdot|\Delta|^{* * *}$ & $\cdot /{ }^{\prime} D$ & . &.$/{ }^{\prime} \Delta$ & $\cdot|\Delta|^{*}$ & $\cdot \mid \Delta r^{*}$ & & & & & & & & \\
\hline $\mathrm{NPi}^{(2)}$ & $-\cdot / \wedge \Delta^{* *}$ & $-\cdot / v \Lambda^{* *}$ & $-\cdot / N \vee^{* *}$ & $\cdot / r$ & r &.$/ 9 * *$ & r//. &.$/ T \Lambda$ & $\cdot \mid \wedge e^{* *}$ & & & & & & & & \\
\hline $\mathrm{NP}_{\mathrm{i}}^{(3)}$ & $-\cdot / M^{* *}$ &.$- / v^{* *}$ & $-\cdot / M^{* *}$ &.$/ 19$ &.$/ 1 \wedge$ &.$/ 99^{* * *}$ &.$/ 1 \Lambda$ & $\cdot / M F$ & $\cdot|\wedge|^{* * *}$ & G &.$/ 9 q^{* * *}$ & & & & & & \\
\hline $\mathrm{NP}_{\mathrm{i}}{ }^{4)}$ &.$- / .9^{* *}$ &.$- / V^{* *}$ & $-\cdot / M^{* *}$ & $\cdot|r|$ & $\cdot / r$ &.$/ 9 q^{* *}$ &.$/ T$ & $\cdot / T V$ & $\cdot / \Lambda \mathrm{f}^{* * *}$ & . &.$/ 9 q^{* *}$ & $\cdot / 9 V^{* * *}$ & & & & & \\
\hline TOP &.$/ 9 \Upsilon^{* * *}$ & $\cdot / M^{* *}$ &.$/ 90^{* *}$ &.$- / . \mu$ & -.1 .0 & $-\cdot / M^{* *}$ & -.1 .0 & -.1 .9 &.$- / 99^{* *}$ &.$- / . \varphi^{c}$ & $-\cdot / \Lambda r^{* *}$ & $-\cdot / \wedge \mathrm{V}^{* *}$ & $-\cdot / \wedge 9^{* *}$ & & & & \\
\hline MID &.- .1 .9 &.$- / . \varphi^{5}$ & -.1 .9 &.- .1 .9 &.$- / 1$ & . &.$- / 1$ &.- .1 .9 & $\cdot / \cdot v$ & .1 .0 &.$/ . r$ &.$/ .+$ &.$/ 1 F$ & $-\cdot / \mu$ & & & \\
\hline LOW & ז & . $/ 9 \Upsilon^{* * *}$ &.$/ 9 \gamma^{* *}$ & .111 & $\cdot 1 \cdot 1$ & $-\cdot|\wedge|^{* *}$ & $.1 \cdot 1$ & .1 .9 & $-\cdot / \Delta \Lambda^{* *}$ & /.r & $-\cdot / \Lambda r^{* *}$ & $-\cdot / N \rho^{* *}$ & $-\cdot / \wedge e^{* *}$ &.$/ 19^{* * *}$ &.$/ 11$ & & \\
\hline$\sigma_{\mathrm{r}}$ & $\cdot 1 \cdot 1$ & $\cdot|r|$ & $\cdot / \cdot V$ &.$/ 98^{* *}$ & $1 / .{ }^{* *}$ & $\cdot / T V$ & $1 / .{ }^{* *}$ &.$/ 9 q^{* *}$ & $\cdot \mid \& \phi^{* *}$ &.$/ \$ \Delta$ & r &.$/ 11$ & $\cdot / r$ &.$- / .0$ &.$- / 1$ & $.1 \cdot 1$ & \\
\hline$\sigma_{\mathrm{my}}$ &.$/ 99^{* *}$ & $\cdot / 9 \gamma^{* *}$ &.$/ 99^{* *}$ &.$/ 1 F$ & .1 .9 & $-\cdot \mid \wedge \varphi^{* *}$ & .1 .9 & $.1 . r$ &.$-|9|^{*}$ &.$/ \cdot V$ & $-\cdot / \wedge \Delta^{* *}$ & $-\cdot / \wedge \mathrm{V}^{* *}$ & $-\cdot / \Lambda \varsigma^{* *}$ &.$/ q \varphi^{* * *}$ & .1 .9 & $\cdot / 9 \Delta^{* *}$ & .1 .9 \\
\hline
\end{tabular}


يارامترها داراى مفهوم ديناميك (زراعى) بايدارى هستند. گروه دوم (GII) شامل معيارهاى (GP

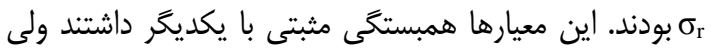

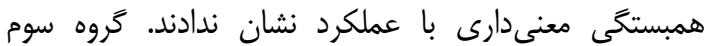

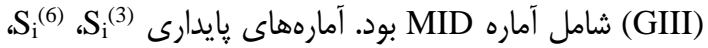

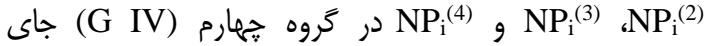

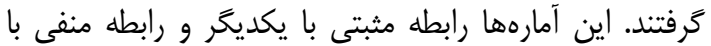

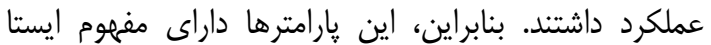

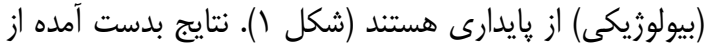

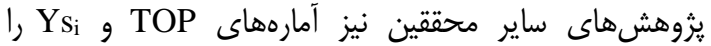

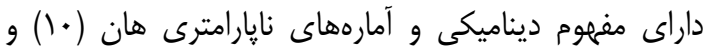

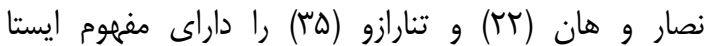

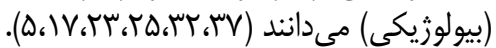

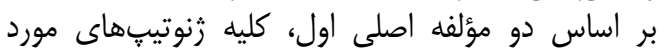

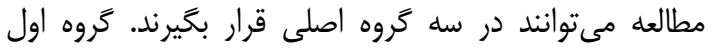

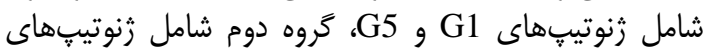

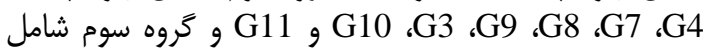

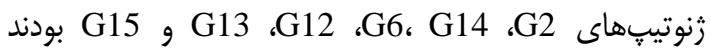

تجزيه به مؤلفهاهاى اصلى بر اساس ميانكَين عملكرد غده و آمارههاى يايدارئ نائه نايار امترى

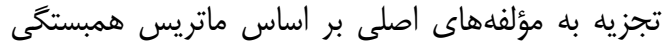

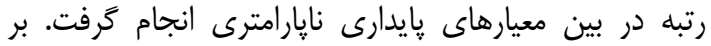

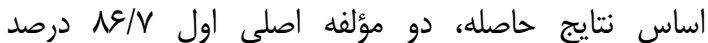

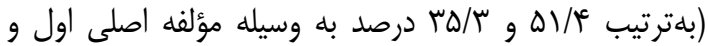

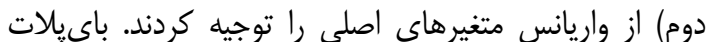

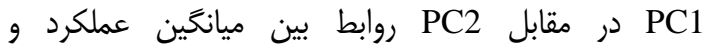

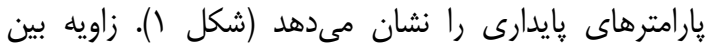

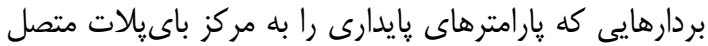

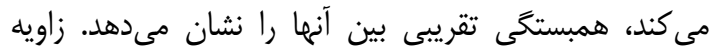

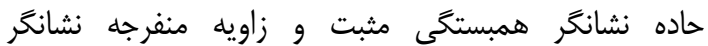

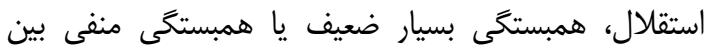

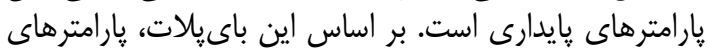

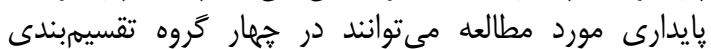

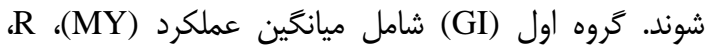

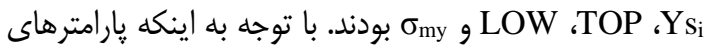

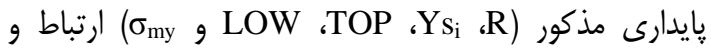
همبستخى مثبتى با ميانكين عملكرد داشتند بنابراين اين إنباط

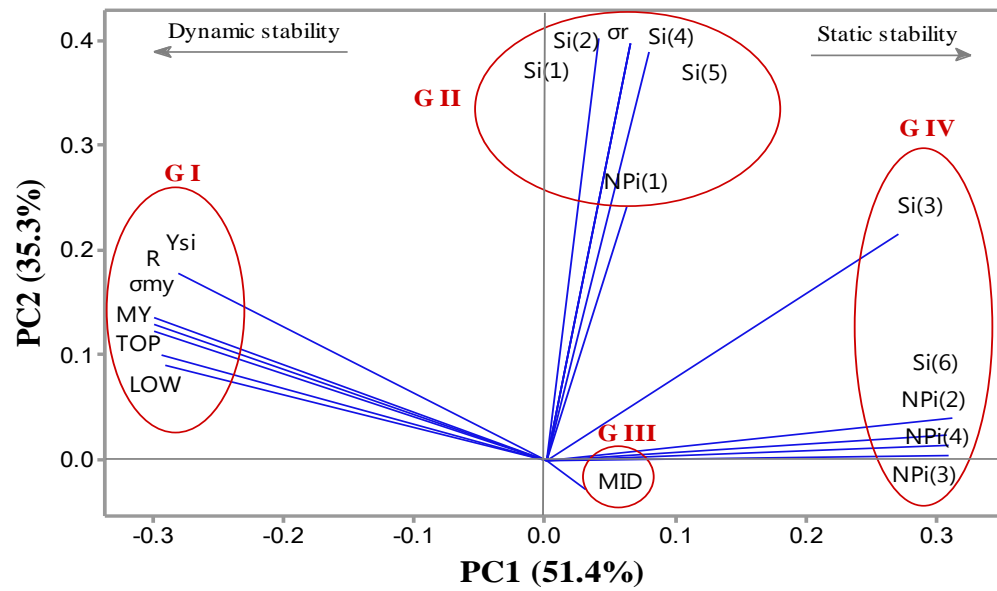

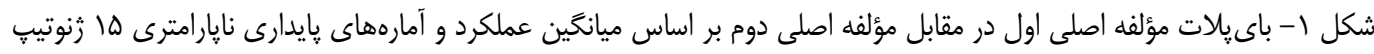
سيبزمينى در شش محيط ميط الفين

Figure 1. Biplot (PC1 vs. PC2) of non-parametric stability procedures based on the rank correlation matrix with yield in 15 potato genotypes in six environments 


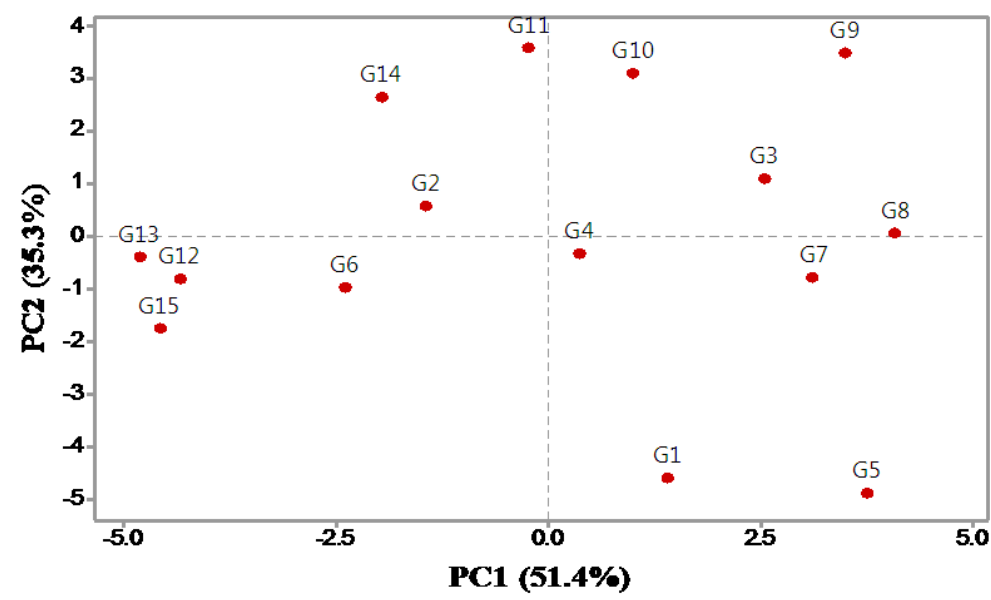

شكل r- باى يلات مؤلفه اصلى اول در مقابل مؤلفه اصلى دوم براى ها زنوتيب سيبزمينى در شش محيط بر اساس ميانخين عملكرد و

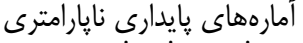

Figure 2. Biplot of PC1 vs. PC2 for 15 potato genotypes in six environments based on mean yield and non-parametric stability procedures

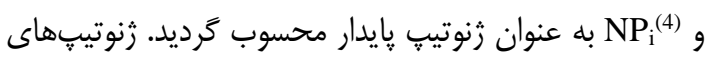

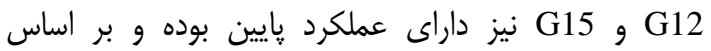
آمارههاى

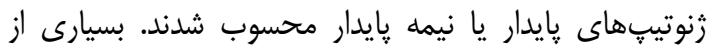

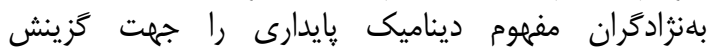

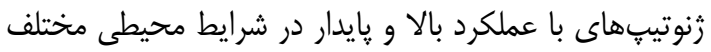

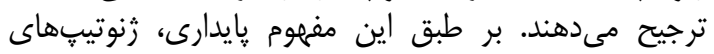

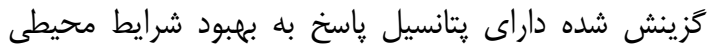

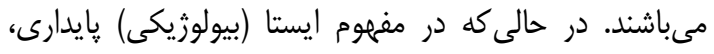

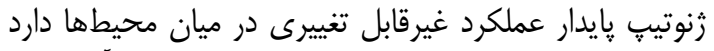

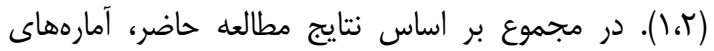

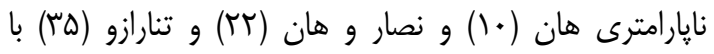

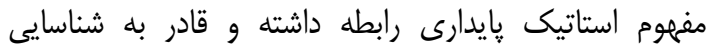

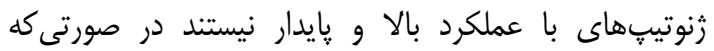

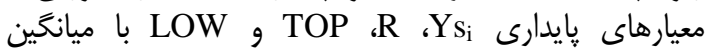

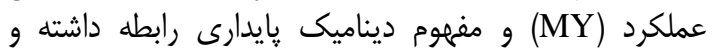

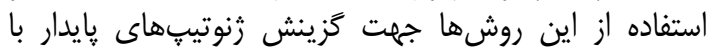

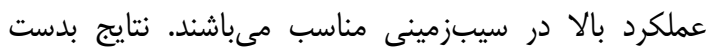

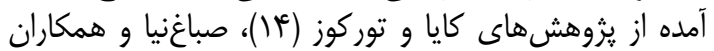

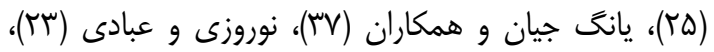

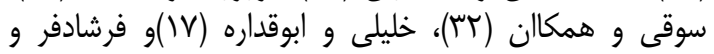

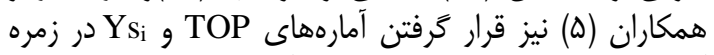

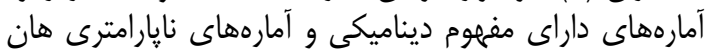

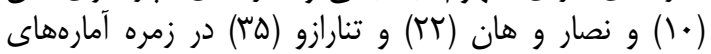

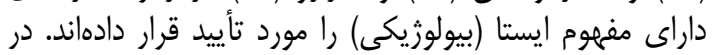

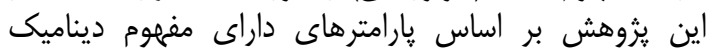

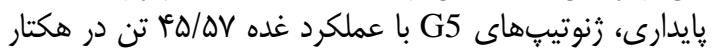

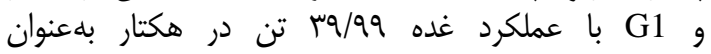

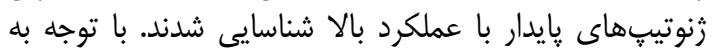

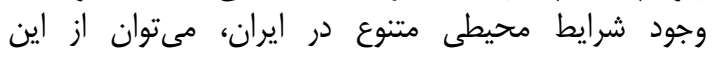

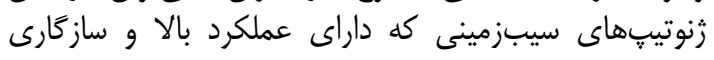

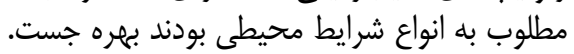

تجزيه كلاستر آمارههاى پايدارى نايارامترى و ميانكَين عملكرد غده تهزه

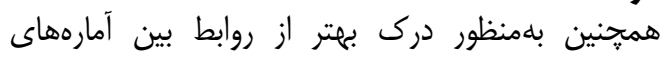

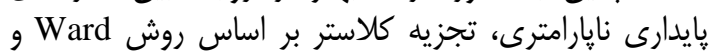

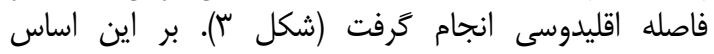

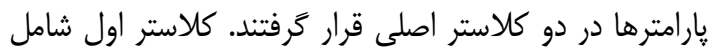

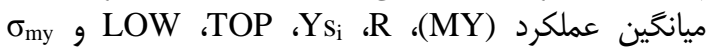
بودند. كلاستر دوم شامل سه زيركلانين

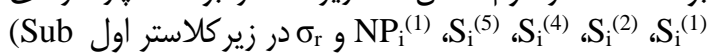

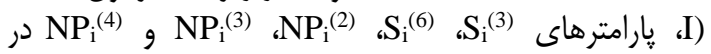
زير كلاستر دوم و يارامتر MID در زير كلاستر سوم (Sub III) جاى گرفت.

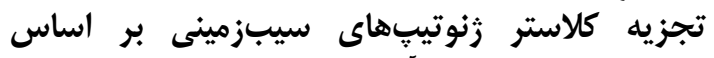

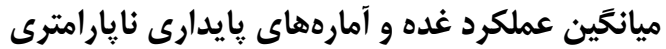

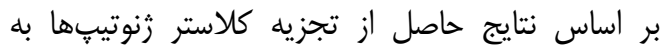

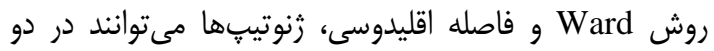

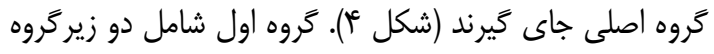

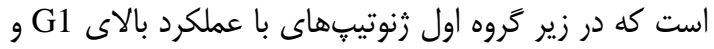

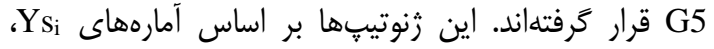

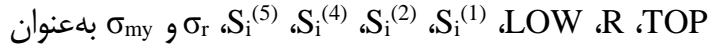

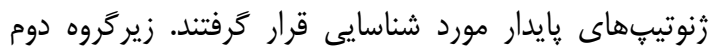

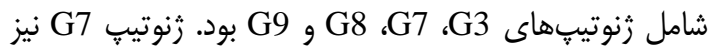

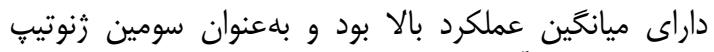
يايدار بر اساس آمارههاى YSi و

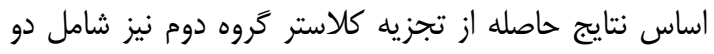

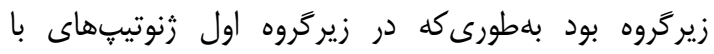

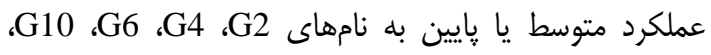

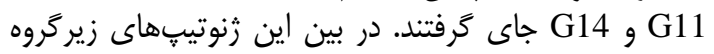

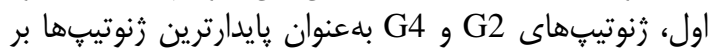

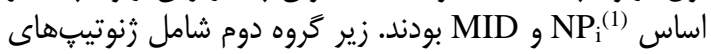

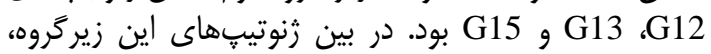

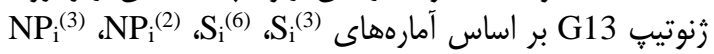




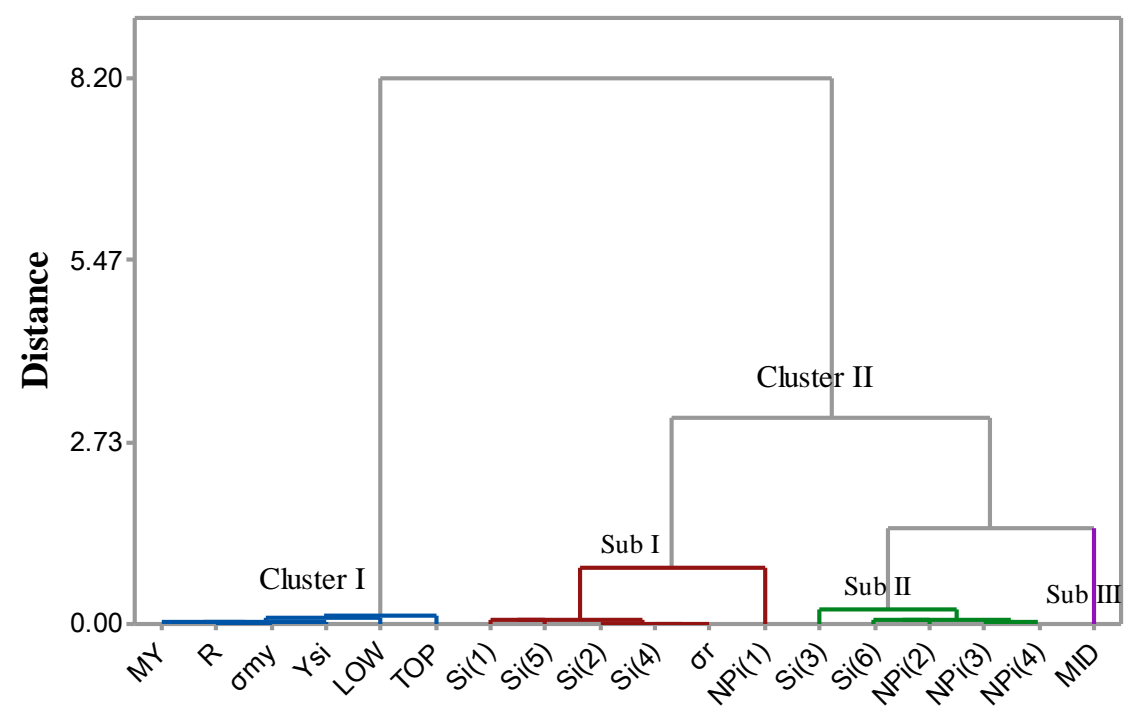

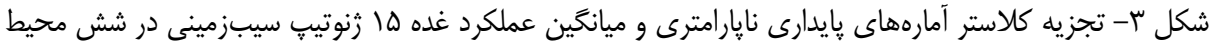

Figure 3. Cluster analysis of non-parametric stability procedures and mean yield of 15 potato genotypes in six environments

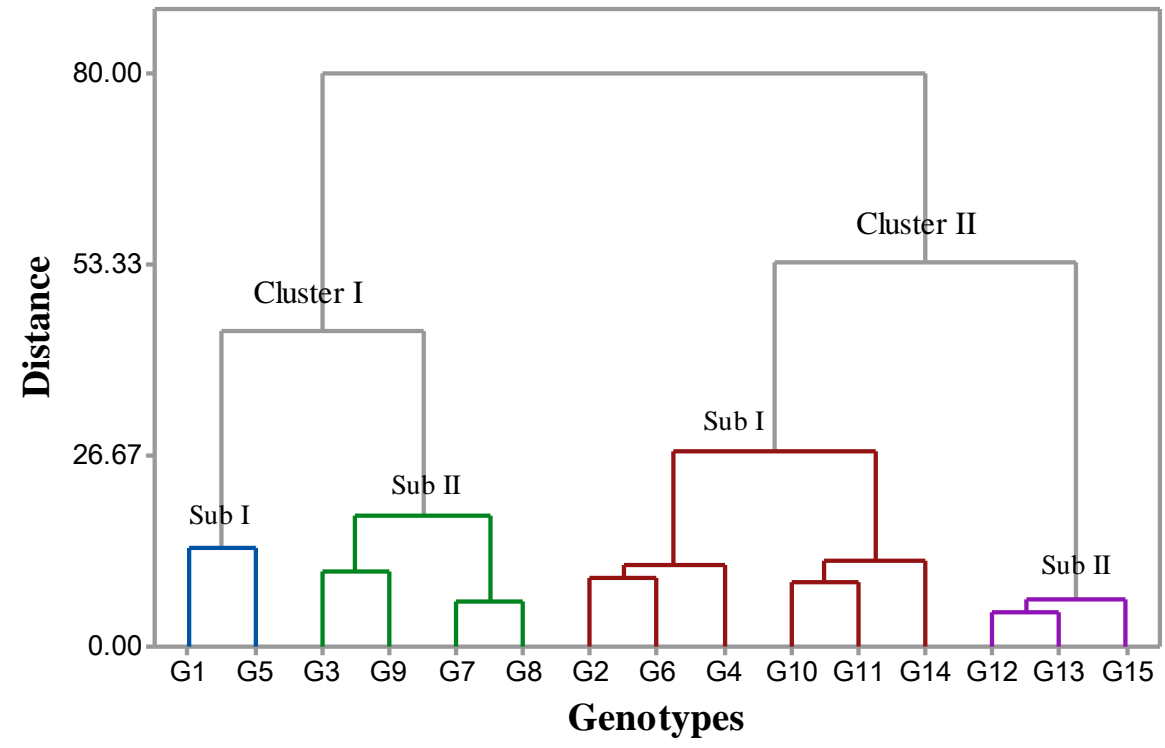

شكل عأ- تجزيه كلاستر ها زنوتيب سيبزمينى بر اساس ميانگَين عملكرد و آمارههاى بايدارى نإِارامترى

Figure 4. Cluster analysis of 15 potato genotypes based on mean yield and non-parametric measures of stability 
1. Becker, H.C. 1981. Correlations among some statistical measures of phenotypic stability. Euphytica, 30: $835-840$.

2. Becker, H.C. and J. Leon. 1988. Stability analysis in plant breeding. Plant Breeding, 101: 1-23.

3. Ebadi Segherloo, A., S.H. Sabaghpour, H. Dehghani and M. Kamrani. 2008. Non-parametric measures of phenotypic stability in chickpea genotypes (Cicer arietinum L.). Euphytica, 162: 221229.

4. FAO. 2008. International year of the potato 2008. Available at www.potato2008.org (accessed 19 August, 2014). Food and Agriculture Organization, Rome.

5. Farshadfar, E., S.H. Sabaghpour and H. Zali. 2012. Comparison of parametric and non-parametric stability statistics for selecting stable chickpea (Cicer arietinum L.) genotypes under diverse environments. Australian Journal of Crop Science, 6: 514-524.

6. Fox, P.N., B. Skovmand, B.K. Thompson, H.J. Braun and R. Cormier. 1990. Yield and adaptation of hexaploid spring triticale. Euphytica, 47: 57-64.

7. Hassanpanah, D. and S.H. Azizi Chakherchaman. 2010. Estimating rank and stability analysis of potato cultivars by nonparametric stability analysis. Research Journal of Environmental Sciences, 4: 173-179.

8. Hassanpanah, D. and H. Hassanabadi. 2011. Evaluation of quantitative and qualitative characteristics of promising potato clones in Ardabil region, Iran. Modern Science of Sustainable Agriculture Journal, 7: 37-48 (In Persian).

9. Hassanpanah, D. and H. Hassanabadi. 2012. Evaluation of quantitative, qualitative and tuber yield stability of 18 promising potato clones in Ardabil province. Journal of Crop Ecophysiology, 8: 219234 (In Persian).

10. Huehn, M. 1979. Beitrage zur Erfassung der phänotypischen Stabilitä t. I. Vorschlag einiger auf Ranginformationen beruhenden Stabilitä tsparameter. EDV in Medizin ünd Biologie, 10: 112-117.

11. Huehn, M. 1990. Nonparametric measures of phenotypic stability. Part 1: Theory. Euphytica, 47: 189194.

12. Huehn, M. 1996. Non-parametric analysis of genotypexenvironment interactions by ranks, In M.S. Kang and H.G. Gauch (ed.) Genotype by environment interaction. CRC Press, BocaRaton, FL, 213228.

13. Kang, M.S. 1988. A rank-sum method for selecting highyielding, stable corn genotypes. Cereal Research Communications, 19: 361-364.

14. Kaya, Y. and M. Turkoz. 2016. Evaluation of genotype by environment interaction for grain yield in durum wheat using non-parametric stability statistics. Turkish Journal of Field Crops, 21:51-59.

15. Ketata, H. 1988. Genotype environment interaction. Proceeding of biometrical technique for cereal breeders. ICARDA. Aleppo. Syria, 16-32.

16. Ketata, H., S.K.M. Yan and M. Nachit. 1989. Relative consistency performance across environments. Int. Symposium on Physiology and Breeding of Winter Cereals for stressed Mediterranean Environments. Montpellier, July, 3-6.

17. Khalili, M. and A. Pour-Aboughadareh. 2016. Parametric and non-parametric measures for evaluating yield stability and adaptability in barley doubled haploid lines. Journal of Agricultural Science and Technology, 18: 789-803.

18. Lachman, J., K. Hamouz, M. Orsak and V. Pivec. 2001. Potato glycoalkaloids and their significance in plant protection and nutrition. Rost Vyroba, 47: 181-1912.

19. Mohammadi R. and A. Amri. 2008. Comparison of parametric and non-parametric methods for selecting stable and adapted durum wheat genotypes in variable environments. Euphytica, 159:419432.

20. Mohammadi, R., A. Abdulahi, R. Haghparast and M. Armion. 2007. Interpreting genotype $x$ environment interactions for durum wheat grain yields using non-parametric methods. Euphytica, 157:239-251.

21. Mut, Z., A. Gülümser and A. Sirat. 2010. Comparison of stability statistics for yield in barley (Hordeum vulgare L.). African Journal of Biotechnology, 9: 1610-1618.

22. Nassar, R. and M. Huhn. 1987. Studies on estimation of phenotypicstability: Tests of significance for non-parametric measures of phenotypic stability. Biometrics, 43: 45-53.

23. Noruzi, E. and A. Ebadi. 2015. Comparison of parametric and non-parametric methods for analysing genotypex environment interactions in sunflower (Helianthus annиus L.) inbred lines. Jordan Journal of Agricultural Sciences, 11: 959-979.

24. Roostaei, M., R. Mohammadi and A. Amri. 2015. Rank correlation among different statistical models in ranking of winter wheat genotypes. The Crop Journal, 2: 154-163.

25. Sabaghnia, N., H. Dehghani and S.H. Sabaghpour. 2006. Nonparametric methods for interpreting genotype $\times$ environment interaction of lentil genotypes. Crop Science, 46: 1100-1106.

26. Safavi, S.M. and S. Bahraminejad. 2017. The evaluation of genotype $\times$ environment interactions for grain yield of oat genotypes using AMMI model. Journal of Crop Breeding, 922: 125-132 (In Persian).

27. Scapim, C.A., V.R. Oliveira, A.L. Braccini, C.D. Cruz, C.A.B. Andrade and C.G.M. Vidigal. 2000 Yield stability in maize (Zea mays L.) and correlations among the parameters of the Eberhart and Russell, Lin and Binns and Huehn models. Genetics and Molecular Biology, 23: 387-393.

28. Secor, G.A. and N.C. Gudmestad. 1999. Managing fungal diseases of potato. Canadian Journal of Plant Pathology, 21: 213-221.

29. Shukla, G.K. 1972. Some statistical aspects of partitioning genotype-environmental components ofvariability. Heredity, 29: 237-242. 
30. Sohrabi, S.S., H. Dehghani and B. Alizadeh. 2016. Evaluation of seed yield stability of promising winter rapeseed (Brassica napus L.) lines using principal coordinates analysis. Journal of Crop Breeding, 8: 152-158 (In Persian).

31. Solomon, K.F., H.A. Smit, E. Malan and W.J. Du Toit. 2007. Comparison study using rank based nonparametric stability statistics of durum wheat. World Journal of Agricultural Sciences, 3:444-450.

32. Soughi, H.A., N.A. Babaeian Jelodar, G.A. Ranjbar and M.H. Pahlevani. 2016. Simultaneous selection based on yield and yield stability in bread wheat genotypes. Journal of Crop Breeding, 8:119-125 (In Persian).

33. Syukur, M., S. Sujiprihati, R. Yunianti and D.A. Kusumah. 2014. Non paramectric stability analysis for yield of hybrid chili pepper (Capsicum annuum L.) across six different environments. Journal Agronomi Indonesia, 42: 32-38.

34. Tadege, M.B., H.Z. Utta and A.A. Aga. 2014. Association of statistical methods used to explore genotypexenvironment interaction (GEI) and cultivar stability. African Journal of Agricultural Research, 9: 2231-2237.

35. Thennarasu, K. 1995. On certain non-parametric procedures for studying genotype environment interactions and yield stability. Ph.D. theses, P.J. School, IARI., New Delhi, $255 \mathrm{pp}$.

36. Truberg, B. and M. Huehn. 2000. Contribution to analysis of genotype by environment interactions: Comparison of different parametric and non-parametric tests for interactions with emphasis on crossover interactions. Agronomy Crop Science, 185: 267-274.

37. Yong-jian, L., D. Chuan, T. Meng-liang, H.U. Er-liang1 and H. Yu-bi. 2010. Yield stability of maize hybrids evaluated in maize regional trials in southwestern China using nonparametric methods. Agricultural Sciences in China, 9: 1413-1422.

38. Zali, H., E. Farshadfar and S.H. Sabaghpour. 2011. Non-parametric analysis of phenotypic Stability in chickpea (Cicer arietinum L.) genotypes in Iran. Crop Breeding Journal, 1: 89-100. 


\title{
Non-Parametric Stability Analysis of Tuber Yield in Potato (Solanum tuberosum L.) Genotypes
}

\author{
Mina Moghaddaszadeh ${ }^{1}$, Rasool Asghari Zakaria ${ }^{2}$ Davoud Hassanpanah $^{3}$ and \\ Nasser Zare ${ }^{4}$ \\ 1 and 4- PhD. Student and Associated Professor, Faculty of Agriculture and Natural Resources, University of \\ Mohaghegh Ardabili, Ardabil, I.R. Iran \\ 2- Faculty of Agriculture and Natural Resources, University of Mohaghegh Ardabili, Ardabil, I.R. Iran, \\ (Corresponding author: r-asghari@uma.ac.ir) \\ 3- Research Staff, Horticulture Crops Research Department, Ardabil Agricultural and Natural Resources Research \\ Centre, AREEO, Ardabil, Iran \\ Received: October 3, $2017 \quad$ Accepted: January 13, 2018
}

\begin{abstract}
In order to study tuber yield stability of 15 potato genotypes, this research was carried out using randomized complete block design with three replications in three different research stations in Iran during two growing seasons. The combined analysis of variance indicated that the main effects of genotype (G), environment (E) and their interactions genotype and environmen $(\mathrm{G} \times \mathrm{E})$ were highly significant $(\mathrm{p}<0.01)$. The principal component analysis (PCA) based on rank correlation matrix indicated that the first two PCAs explained $86.7 \%$ of the variance of original variables. Based on bi-plot analysis, the stability parameters were classified into four groups. Clustering of the genotypes according to the mean yield and nonparametric stability statistics showed that there were two main clusters. Overall, according to mean rank of nonparametric stability parameters, G1, G15, G5, G6, G12 and G13 had the lowest variations and were recognized as the most stable genotypes. Genotypes G9, G11, G14, G3 and G7 had the highest values of mean rank of parameters and therefore, would be considered to be the most unstable. According to the present study, the stability measures $\mathrm{Ys}_{\mathrm{i}}, \mathrm{R}$, TOP and LOW were associated with mean yield (MY) and the dynamic concept of stability. Therefore, these procedures were suitable for selecting stable and high yielding genotypes. Based on these parameters, genotypes G5 (45.57 t/ha) and G1 (39.99 t/ha) were identified as high yield stable genotypes.
\end{abstract}

Keywords: Cluster analysis, Potato, Principal component analysis, Stability analysis 